\title{
Ancient DNA analysis of 101 cattle remains: limits and prospects
}

\author{
Ceiridwen J. Edwards ${ }^{a}$, David E. MacHugh ${ }^{\mathrm{b}}$, Keith M. Dobney ${ }^{\mathrm{c}}$, Louise Martin ${ }^{\mathrm{d}}$, \\ Nerissa Russell $^{\mathrm{e}}$, Liora K. Horwitz ${ }^{\mathrm{f}}$, Susan K. McIntosh ${ }^{\mathrm{g}}$, Kevin C. MacDonald ${ }^{\mathrm{h}}$, \\ Daniel Helmer ${ }^{\mathrm{i}}$, Anne Tresset ${ }^{\mathrm{j}}$, Jean-Denis Vigne ${ }^{\mathrm{j}}$, Daniel G. Bradley ${ }^{\mathrm{a} *}$ \\ ${ }^{a}$ Department of Genetics, Trinity College, Dublin 2, Ireland \\ ${ }^{\mathrm{b}}$ Department of Animal Science and Production and Conway Institute of Biomolecular and Biomedical Research, Faculty of Agriculture, \\ University College Dublin, Belfield, Dublin 4, Ireland \\ ${ }^{c}$ Department of Archaeology, University of Durham, South Road, Durham DH1 3LE, UK \\ ${ }^{\mathrm{d}}$ Institute of Archaeology, University College London, 31-34 Gordon Square, London WC1H OPY, UK \\ ${ }^{\mathrm{e}}$ Department of Anthropology, Cornell University, Ithaca, NY 14853, USA \\ ${ }^{\mathrm{f}}$ Department of Evolution, Systematics and Ecology, The Hebrew University, Berman Building, Givat Ram, Jerusalem, Israel \\ ${ }^{\mathrm{g}}$ Department of Anthropology, Rice University, 6100 Main Street, Houston, TX, USA \\ ${ }^{\mathrm{h}}$ School of Oriental and African Studies, University of London, Thornhaugh Street, Russell Square, London WC1H OXG, UK \\ ${ }^{\mathrm{i}}$ CNRS, GREMMO-MOM, IPO Jalès, 07460 Berrias, France \\ ${ }^{\mathrm{j}}$ CNRS Muséum National d'Histoire Naturelle, Archéozoologie et Histoire des Sociétés (ESA8045), Bâtiment d'Anatomie Comparée, \\ 55 Rue Buffon, Paris, France
}

Received 7 April 2003; received in revised form 21 October 2003; accepted 12 November 2003

\begin{abstract}
A total of 101 cattle teeth and bones from 13 archaeological sites between 1000 to 9000 years old were assessed for the presence of verifiable mitochondrial sequences. It was possible to reproducibly amplify and sequence mitochondrial control region DNA extracted from twelve of the samples. The results were compared with published extant data by constructing phylogenetic networks. The sequences obtained from the cattle specimens were either identical to the reference sequence for modern cattle or closely related to it. They included three sequences not previously documented. The network analysis of the ancient data highlights the proximity of the ancient DNA cattle sequences to modern Near Eastern, European and African Bos taurus, as well as regional continuity. (C) 2003 Elsevier Ltd. All rights reserved.
\end{abstract}

Keywords: Ancient DNA; Cattle; Biomolecular archaeology; Mitochondrial haplotypes; Polymerase chain reaction

\section{Introduction}

\subsection{Patterns of mitochondrial DNA diversity in Bos taurus}

Ancient and extant mitochondrial DNA (mtDNA) diversity in cattle shows geographical structure that can be related to the process of domestication [47]. Three divergent families of sequences have been described: (1) a South Asian group, which was incorporated by domestication of the $B$. indicus progenitor; (2) those found in modern B. taurus and believed to originate

\footnotetext{
* Corresponding author. Tel.: +353-1-608-1088; fax: +353-1-679-8558

E-mail address: dbradley@tcd.ie (D.G. Bradley).
}

from Western Asia; and (3) a separate clade that to-date has only been encountered in six wild aurochs $(B$. primigenius) bones sampled in Britain $[2,47]$. Examples of each are given in Table 1.

Analyses of previously described 536 B. taurus mtDNA sequences indicate that they all root to the taurine phylogeny through one of five common 240 base pair (bp) haplotypes $[6,9,27,32,33,47]$. These haplotypes are designated as $\mathrm{T}, \mathrm{T} 1, \mathrm{~T} 2, \mathrm{~T} 3$ and $\mathrm{T} 4$, and represent five geographically distributed clusters. African diversity is almost exclusively described by haplogroup T1, and this group is found only at low frequencies elsewhere [47]. Haplogroup T4 has thus far only been detected in Japanese cattle [33]. The other three variants occur at appreciable frequencies in the Near East, with one of 
Table 1

Mitochondrial control region sequence variations

\begin{tabular}{|c|c|c|c|c|c|c|c|c|c|c|c|c|c|c|c|c|c|c|c|c|c|c|c|c|c|c|}
\hline & 1 & 1 & 1 & 1 & 1 & 1 & 1 & 1 & 1 & 1 & 1 & 1 & 1 & 1 & 1 & 1 & 1 & 1 & 1 & 1 & 1 & 1 & 1 & 1 & 1 & Assignment to \\
\hline & 6 & 6 & 6 & 6 & 6 & 6 & 6 & 6 & 6 & 6 & 6 & 6 & 6 & 6 & 6 & 6 & 6 & 6 & 6 & 6 & 6 & 6 & 6 & 6 & 6 & haplogroup \\
\hline & 0 & 0 & 0 & 0 & 0 & 0 & 0 & 0 & 0 & 0 & 0 & 1 & 1 & 1 & 1 & 1 & 1 & 1 & 1 & 1 & 1 & 1 & 1 & 1 & 1 & \\
\hline & 4 & 4 & 5 & 5 & 5 & 5 & 7 & 8 & 8 & 8 & 9 & 0 & 0 & 1 & 1 & 1 & 1 & 1 & 2 & 2 & 3 & 3 & 3 & 4 & 4 & \\
\hline & 2 & 9 & 0 & 1 & 7 & 8 & 4 & 2 & 4 & 5 & 3 & 2 & 9 & 0 & 3 & 6 & 7 & 9 & 1 & 2 & 0 & 7 & 8 & 3 & 7 & \\
\hline $\begin{array}{l}\mathrm{T} / \mathrm{T} 3 \\
\text { consensus }\end{array}$ & $\mathrm{T}$ & $\mathrm{C}$ & $\mathrm{C}$ & $\mathrm{T}$ & G & $\mathrm{C}$ & $\mathrm{T}$ & G & $\mathrm{C}$ & $\mathrm{T}$ & G & G & $\mathrm{T}$ & $\mathrm{C}$ & $\mathrm{T}$ & $\mathrm{T}$ & G & $\mathrm{T}$ & G & $\mathrm{T}$ & $\mathrm{T}$ & $\mathrm{T}$ & $\mathrm{T}$ & A & $\mathrm{T}$ & - \\
\hline $\mathrm{T} 1$ consensus & . & . & $\mathrm{T}$ & . & . & . & . & . & . & . & . & . & . & . & $\mathrm{C}$ & . & . & . & . & . & . & . & . & . & . & - \\
\hline $\mathrm{T} 2$ consensus & . & . & . & . & $\mathrm{C}$ & . & . & . & . & . & . & . & . & . & . & . & . & . & . & . & . & . & . & . & . & - \\
\hline $\mathrm{T} 4$ consensus & $\mathrm{C}$ & . & . & . & . & . & . & . & . & . & A & . & . & . & . & . & . & . & . & . & . & . & . & . & . & - \\
\hline $\begin{array}{l}\text { British } \\
\text { aurochs }\end{array}$ & . & $\mathrm{T}$ & . & $\mathrm{C}$ & . & $\mathrm{T}$ & $\mathrm{C}$ & . & . & $\mathrm{C}$ & . & . & & . & . & . & . & . & . & $\mathrm{C}$ & . & . & . & . & . & - \\
\hline CH11 & . & . & . & . & . & . & . & . & . & . & . & . & . & . & . & . & . & . & . & . & . & . & . & . & . & T/T3 \\
\hline ТВ03 & . & . & $\mathbf{T}$ & . & . & . & C & . & . & . & . & . & . & . & . & . & . & . & . & . & . & . & . & . & . & $\mathbf{T}$ \\
\hline TB07 & . & . & . & . & . & . & . & . & . & . & . & . & . & . & . & . & . & . & . & . & . & . & . & . & . & T/T3 \\
\hline Ardèche 01 & . & . & . & . & . & . & . & . & . & . & . & . & . & . & . & . & . & . & . & . & . & . & . & . & . & T/T3 \\
\hline Ardèche 02 & . & . & . & . & . & . & . & . & . & . & . & . & . & . & . & . & . & . & . & . & . & . & . & . & . & T/T3 \\
\hline Bercy 09 & . & . & . & . & . & . & . & . & . & . & . & . & . & . & . & . & . & . & . & . & . & . & . & . & . & T/T3 \\
\hline Bercy 10 & . & . & . & . & C & . & . & . & . & . & . & . & . & . & . & . & . & . & . & . & . & . & . & . & . & T2 \\
\hline Bercy 13 & . & . & . & . & . & . & . & . & $T$ & . & . & . & . & $T$ & . & . & . & . & . & . & . & . & . & . & . & T3 \\
\hline Africa $05 c$ & . & . & $T$ & . & . & . & . & . & . & C & . & . & . & . & $\mathrm{C}$ & . & . & . & . & . & . & . & . & . & . & T1 \\
\hline Africa 05d & . & . & $T$ & . & . & . & . & . & . & $\mathrm{C}$ & . & . & . & . & C & . & . & . & . & . & . & . & . & . & . & T1 \\
\hline Africa 06 & . & . & . & . & . & . & . & . & . & . & . & . & . & . & . & . & . & . & . & . & . & . & . & . & . & T/T3 \\
\hline Africa 07 & . & . & $\mathrm{T}$ & . & . & . & C & . & . & C & . & . & C & . & C & . & . & . & . & . & . & . & . & . & . & T1 \\
\hline B. indicus & . & $\mathrm{T}$ & . & . & A & $\mathrm{T}$ & C & A & $\mathrm{T}$ & C & . & A & $\mathrm{C}$ & & $\mathrm{C}$ & $\mathrm{C}$ & A & C & A & $\mathrm{C}$ & $\mathrm{C}$ & $\mathrm{C}$ & $\mathrm{C}$ & $*$ & C & - \\
\hline
\end{tabular}

The variable positions in control region sequences of archaeological cattle samples aligned to the European consensus haplotype (T3). Differences are indicated, with a period (.) denoting identity. Sequence codes are given in the first column and only variable sites are shown. The sequence positions from the BOVMT GENBANK sequence are given above each column (accession number V00654; [1]). The common and putatively ancestral Middle Eastern and European consensus sequence is denoted T/T3 (see text), with T1, T2 and T4 denoting the consensus sequences from Africa, the Middle East/Europe, and Japan respectively. The putative ancestral British aurochs sequence is also included (GENBANK accession number AF336746; [47]), as well as a representative $B$. indicus sequence (HA2; [6]). All ancient sequences generated as part of this study are shown in bold type, and each sample has been assigned to one of the six main haplogroups by means of its relative position in the median networks. 
these, T3, predominating across Europe. B. taurus mtDNA sequence diversity is highest in Near Eastern cattle populations and this, along with the phylogenetic distinction of British aurochs sequences, has been used to argue against a local origin for Western European cattle. However, the African and East Asian-specific $B$. taurus variants may reflect regional incorporation of regional $B$. primigenius strains. Interestingly, haplotypes in all regions show star-like patterns surrounding some or all of the main ancestral sequences (T, T1, T2 and T3), which may be argued as being characteristic of past population expansions, here likely to be associated with the domestication process.

Prior data is one advantage of studying ancient mtDNA in cattle. Also, the mtDNA molecule is present at a high copy number in most somatic cells (approximately 1000 copies), and thus the survival and recovery of intact mtDNA fragments is more likely than that of single-copy nuclear DNA. In addition, the segment of the D-loop analysed here is the most variable region of the mitochondrial genome. The $157 \mathrm{bp}$ section amplified in these ancient specimens contains 66 sites that vary among aurochs and modern B. taurus and B. indicus cattle, with the larger $176 \mathrm{bp}$ section defining a further 31 sites $[2,6,47]$.

\subsection{Ancient DNA-criteria for rating success or failure}

Several researchers in the field have set out various criteria to measure the authenticity of ancient (aDNA) results (for example [8,31]). We believe the most important criterion to be reproducibility. If the sequence data obtained is to be accepted as authentic then it must be reproducible. In our opinion, this condition is fulfilled when consistent sequences are obtained in three or more amplifications, with at least one from each of two separate extractions. We use a physically isolated preamplification work area to avoid carry-over contamination, with several additional control amplifications to detect low-copy number DNA. As shorter length fragments are easier to amplify from ancient samples than longer ones [2,30], we target small fragments using species-specific primers, as suggested by Richards et al. [37]. Extractions and amplification reactions are run in tandem with ancient specimens from distantly related species for which the primers should not work [45]. All sequence data obtained has to be phylogenetically consistent before the results are accepted as genuine.

\subsection{Archaeological site information}

The material tested here vary in date, from a Chalcolithic-Bronze Age site in Syria, Neolithic sites in Anatolia, Israel and France, and Iron Age sites from Western Africa. It also varies in macroclimatic condition, from semi-arid Near Eastern and African areas, to wet (Southern France) and temperate (Paris Basin) Mediterranean regions. Thirteen sites were sampled: seven from the Near East (Çatalhöyük in Anatolia; five sites around Israel-Tel Hreiz, Atlit Yam, Newe Yam, Kfar Hahoresh and Abu Gosh; and Tell Brak in Syria); three from France (two in the Ardèche region-Baume d'Oullen and Combe Obscure; and one in Paris-Bercy); and three sites from Western Africa (Jenné-jeno and Kaniana in Mali; plus Sincu Bara in Senegal). These sites also display different conditions of deposit, from settlement mounds (African and most NearEastern sites) to karstic caves (Ardèche), and to waterlogged areas (Bercy, Paris; Tel Hreiz, Atlit Yam and Newe Yam, Israel). Details of these sites and associated information are shown in Table 2.

\section{Methods}

\subsection{Archaeological material collection}

101 Bos sp. teeth and bones were retrieved from various archaeological sites (Table 2). Based on archaeological context, 23 Chalcolithic-Bronze Age cattle samples were extracted, alongside 62 Neolithic and 16 Iron Age samples. In addition, three Equus sp. (horse) teeth from Çatalhöyük, three Sus scrofa (pig) bones from Israel, and one S. scrofa (wild boar) vertebra from the 5000 year-old site of Carsington Pasture Cave in Derbyshire, were extracted and used as controls.

Previous studies of aDNA survival have found a good correspondence between bone preservation and the presence of amplifiable endogenous DNA [7,21,37]. Most of the Near Eastern bovine bone material was light in weight, sandy coloured, with damaged external surfaces. The majority of these remains came from settlement mounds in semi-arid areas, apart from those bones from Tel Hreiz, Atlit Yam and Newe Yam, which were all from submerged sites in the Mediterranean Sea. None of the Near Eastern remains could be considered well preserved using the gross morphological criteria outlined by Hagelberg et al. [18]. On the other hand, teeth and bones from the two French caves, Baume d'Oullen and Combe Obscure, were very well preserved and, with reference to radiocarbon dates and previous aDNA data from caves in Southern France [20], could be expected to have both well preserved collagen and aDNA. Bercy is located on the edge of a paleochannel of the Seine [5], and all bones used in this study were taken from areas within fluctuating water levels. Bones from immersed areas were brown-black in colour, and this staining was assumed to mainly be due to humic acids. Stable isotopes analyses carried out previously on these bones have revealed good collagen preservation in the majority (Table 2). Extraction yields were relatively high, with carbon and nitrogen amounts, as well as the $\mathrm{C} / \mathrm{N}$ ratios, being similar to those obtained on modern 
Table 2

Site and amplification details, with associated information

\begin{tabular}{|c|c|c|c|c|c|c|c|c|c|c|}
\hline \multirow[t]{2}{*}{ Lab code } & \multirow[t]{2}{*}{ Achaeological code } & \multirow[t]{2}{*}{ Skeletal pan } & \multirow{2}{*}{$\begin{array}{l}\text { Date } \\
\text { excavated }\end{array}$} & \multirow[t]{2}{*}{ Extra information } & \multicolumn{4}{|c|}{ PCR amplification } & \multirow{2}{*}{$\begin{array}{l}\text { Sequence } \\
\text { cluster(s) }\end{array}$} & \multirow{2}{*}{$\begin{array}{l}\text { Reasons for } \\
\text { success/failure }\end{array}$} \\
\hline & & & & & $\begin{array}{l}\text { No. of } \\
\text { extractions }\end{array}$ & $\begin{array}{l}157 \mathrm{bp} \\
\text { product }\end{array}$ & $\begin{array}{l}176 \mathrm{bp} \\
\text { product }\end{array}$ & $\begin{array}{l}\text { Maximum } \\
\text { sequence } \\
\text { length } \\
\text { (bp) }\end{array}$ & & \\
\hline \multicolumn{11}{|c|}{ Catalhöyük—c 9000-8000 BP (early Neolithic) ([22,34,40]; http://www.catalhoyuk.com) } \\
\hline $\mathrm{CH} 01$ & $\begin{array}{l}\text { CH1996 \#5 Area } \\
\text { South/Unit } 1668\end{array}$ & metatarsal & 1996 & stored at RT & 4 & no & no & - & - & - \\
\hline $\mathrm{CH} 02$ & $\begin{array}{l}\text { CH1996 \#4 Area } \\
\text { South/Unit } 1520\end{array}$ & metacarpal & 1996 & stored at RT & 4 & 1 of 8 & no & 157 & $\mathrm{~T} / \mathrm{T} 3$ & non-reproducible \\
\hline $\mathrm{CH} 03$ & $\begin{array}{l}\text { CH1996 \#3 Area } \\
\text { South/Unit } 1579\end{array}$ & metacarpal & 1996 & stored at RT & 3 & 1 of 6 & no & 107 & $\mathrm{~T} / \mathrm{T} 3$ & non-reproducible \\
\hline $\mathrm{CH} 04$ & $\begin{array}{l}\text { CH1996 \#1 Area } \\
\text { South/Unit } 1832\end{array}$ & metacarpal & 1996 & stored at RT & 2 & 1 of 4 & no & 124 & $\mathrm{~T} / \mathrm{T} 3$ & non-reproducible \\
\hline $\mathrm{CH} 05$ & $\begin{array}{l}\text { CH1996 \#X1 Area } \\
\text { South/Unit } 1816\end{array}$ & metatarsal & 1996 & stored at RT & 2 & no & no & - & - & - \\
\hline $\mathrm{CH} 07$ & $\begin{array}{l}\text { CH1996 \#4 Area } \\
\text { South/Unit } 1579\end{array}$ & metapodial & 1996 & stored in freezer & 4 & 3 of 8 & no & 157 & $\begin{array}{l}\mathrm{T} / \mathrm{T} 3 \\
\text { aurochs }\end{array}$ & contradiction \\
\hline $\mathrm{CH} 08$ & $\begin{array}{l}\text { CH1996 \#8 Area } \\
\text { South/Unit } 1668\end{array}$ & metacarpal & 1996 & stored in freezer & 2 & 1 of 4 & no & 132 & $\mathrm{~T} / \mathrm{T} 3$ & non-reproducible \\
\hline $\mathrm{CH} 09$ & $\begin{array}{l}\text { CHI996 \#3 Area } \\
\text { South/Unit } 1520\end{array}$ & metacarpal & 1996 & stored in freezer & 2 & 3 of 4 & 2 of 4 & 157 & $\begin{array}{l}\mathrm{T} / \mathrm{T} 3 \\
\text { aurochs }\end{array}$ & contradiction \\
\hline $\mathrm{CH} 10$ & $\begin{array}{l}\text { CH1996 \#3 Area } \\
\text { South/Unit } 1822\end{array}$ & metacarpal & 1996 & stored in freezer & 2 & no & no & - & - & - \\
\hline $\mathrm{CH} 11$ & $\begin{array}{l}\text { CH1996 \#X1 Area } \\
\text { North/Unit } 1430\end{array}$ & metacarpal & 1996 & stored in freezer & 3 & 4 of 6 & no & 133 & $\mathrm{~T} / \mathrm{T} 3$ & reproducible \\
\hline CH99-01 & 90 2331.F3 South & tooth & 1997 & CH97-B10 & 2 & no & no & - & - & - \\
\hline CH99-02 & 74 2706.F1 South & tooth & 1997 & CH97-B01 & 2 & no & no & - & - & - \\
\hline CH99-03 & 83 2331.F2 South & tooth & 1997 & CH97-B07 & 2 & no & no & - & - & - \\
\hline CH99-04 & 82 2303.F1 South & tooth & 1997 & CH97-B06 & 2 & no & no & - & - & - \\
\hline CH99-05 & 80 1873.F611 South & tooth & 1997 & CH97-B04 & 2 & no & no & - & - & - \\
\hline CH99-06 & 85 2340.F2 South & tooth & 1997 & CH97-B09 & 2 & no & no & - & - & - \\
\hline CH99-07 & 2845.X8 S.11 (S3) South & tooth & 1998 & & 2 & 2 of 4 & no & 157 & T/T3 & contradiction \\
\hline CH99-08 & 3375.F1 (S6) South & tooth & 1998 & from Plot S2 & 1 & no & no & - & - & - \\
\hline CH99-09 & 2845.X5 (S2) South & tooth & 1998 & & 2 & 1 of 4 & no & 157 & $\mathrm{~T} / \mathrm{T} 3$ & non-reproducible \\
\hline CH99-10 & 3314.F159 South & tooth & 1998 & 1 & no & no & - & - & - & - \\
\hline CH99-11 & 3129.F54 S.3 (S5) South & tooth & 1998 & 1 & no & no & - & - & - & - \\
\hline CH99-12 & 3702.X4 (S7) South & tooth & 1998 & 1 & no & no & - & - & - & - \\
\hline CH99-13 & 2845.X7 S.11 (S4) South & tooth & 1998 & 1 & no & no & - & - & - & - \\
\hline \multicolumn{11}{|c|}{ Israel—c. $9000-6000$ BP (Neolithic) [13-16,24] } \\
\hline IS01 & Tel Hreiz-30/93-24/61 & radius & 1993 & submerged in Mediterranean Sea & 1 & no & no & - & - & - \\
\hline IS02 & Tel Hreiz-30/93-33 & humerus & 1993 & submerged in Mediterranean Sea & 1 & no & no & - & - & - \\
\hline
\end{tabular}




\begin{tabular}{|c|c|c|c|c|c|c|c|c|c|c|}
\hline \multirow[t]{2}{*}{ Lab code } & \multirow[t]{2}{*}{ Achaeological code } & \multirow[t]{2}{*}{ Skeletal pan } & \multirow{2}{*}{$\begin{array}{l}\text { Date } \\
\text { excavated }\end{array}$} & \multirow[t]{2}{*}{ Extra information } & \multicolumn{4}{|c|}{ PCR amplification } & \multirow{2}{*}{$\begin{array}{l}\text { Sequence } \\
\text { cluster(s) }\end{array}$} & \multirow{2}{*}{$\begin{array}{l}\text { Reasons for } \\
\text { success/failure }\end{array}$} \\
\hline & & & & & $\begin{array}{l}\text { No. of } \\
\text { extractions }\end{array}$ & $\begin{array}{l}157 \mathrm{bp} \\
\text { product }\end{array}$ & $\begin{array}{l}176 \mathrm{bp} \\
\text { product }\end{array}$ & $\begin{array}{l}\text { Maximum } \\
\text { sequence } \\
\text { length } \\
\text { (bp) }\end{array}$ & & \\
\hline IS03 & Tel Hreiz-30/93-27 & humerus & 1993 & submerged in Mediterranean Sea & 1 & no & no & - & - & - \\
\hline IS04 & Tel Hreiz-30/93-24/60 & scapula & 1993 & submerged in Mediterranean Sea & 1 & no & no & - & - & - \\
\hline IS05 & Newe Yam-30/93-9/61 & humerus & 1993 & submerged in Mediterranean Sea & 1 & no & no & - & - & - \\
\hline IS06 & $\begin{array}{l}\text { Newe } \\
\text { Yam-28/8/90-5/9/90 }\end{array}$ & pelvis & 1990 & submerged in Mediterranean Sea & 1 & no & no & - & - & - \\
\hline IS07 & Newe Yam-30/93-9/159 & tibia & 1993 & submerged in Mediterranean Sea & 1 & no & no & - & - & - \\
\hline IS08 & Newe Yam-30/90-9 & metatarsal & 1990 & submerged in Mediterranean Sea & 1 & no & no & - & - & - \\
\hline IS09 & Newe Yam-21/94-47 & scapula & 1994 & submerged in Mediterranean Sea & 1 & no & no & - & - & - \\
\hline IS10 & Newe Yam-30/93-9/78 & femur & 1993 & submerged in Mediterranean Sea & 1 & no & no & - & - & - \\
\hline IS11 & Newe Yam-30/90-10/125 & radius & 1990 & submerged in Mediterranean Sea & 1 & no & no & - & - & - \\
\hline IS12 & $\begin{array}{l}\text { Atlit Yam-Bld. } 11 \mathrm{Ht} . \\
0-120\end{array}$ & tibia & 1988 & submerged in Mediterranean Sea & 1 & no & no & - & - & - \\
\hline IS13 & $\begin{array}{l}\text { Atlit Yam-Bld. } 11 \mathrm{Ht} \text {. } \\
60-80\end{array}$ & femur & 1988 & submerged in Mediterranean Sea & 1 & no & no & - & - & - \\
\hline IS14 & $\begin{array}{l}\text { Atlit Yam-Bld. } 7 \text { No. } \\
14\end{array}$ & scapula & 1990 & submerged in Mediterranean Sea & 1 & no & no & - & - & - \\
\hline IS15 & Atlit Yam—Bld. 9 No. 9 & tibia & 1990 & submerged in Mediterranean Sea & 1 & no & no & - & - & - \\
\hline IS16 & $\begin{array}{l}\text { Atlit Yam-Bld. } 9 \text { No. } \\
47\end{array}$ & tibia & 1990 & submerged in Mediterranean Sea & 1 & 1 of 2 & no & 157 & aurochs & mosaic \\
\hline IS17 & AtlitYam-Bld. 20 & femur & 1989 & submerged in Mediterranean Sea & 1 & no & no & - & - & - \\
\hline IS18 & $\begin{array}{l}\text { Kfar Hahoresh—L1005 } \\
\text { No. } 230\end{array}$ & tibia & 1992 & 1 & no & no & - & - & - & - \\
\hline IS19 & Kfar Hahoresh—L1005 & metatarsal & 1992 & 1 & no & no & - & - & - & - \\
\hline IS20 & $\begin{array}{l}\text { Kfar Hahoresh-ZW58d } \\
\text { 45/3.88-3.98 }\end{array}$ & radius & 1997 & 1 & no & no & - & - & - & - \\
\hline IS21 & $\begin{array}{l}\text { Kfar Hahoresh-Q57a } \\
\text { 30/4,012/30 L1155 }\end{array}$ & metatarsal & 1997 & 1 & no & no & - & - & - & - \\
\hline IS 22 & $\begin{array}{l}\text { Kfar Hahoresh-L1005 } \\
\text { No. } 153\end{array}$ & tibia & 1992 & 1 & no & no & - & - & - & - \\
\hline IS 23 & $\begin{array}{l}\text { Abu Gosh-L146 AA5 } \\
\text { Level } 3 \text { Ht. 696.02-695.96 }\end{array}$ & scapula & 1995 & 1 & no & no & - & - & - & - \\
\hline \multicolumn{11}{|c|}{ Tell Brak, Syria—c. 4000-2000 BC (Chalcolithic-Bronze Age) [10] } \\
\hline TB01 & TB94 A1186:2/HS & limb bone & 1994 & 4th-3rd millennium & 2 & 1 of 4 & no & 157 & $\mathrm{~T} / \mathrm{T} 3$ & non-reproducible \\
\hline ТВ02 & TB94 A2047:2/HS & bone & 1994 & 4th-3rd millennium & 2 & no & no & - & - & - \\
\hline ТВ03 & TB94 A1077:2/HS & vertebra & 1994 & 4th-3rd millennium & 2 & 4 of 4 & no & 157 & $\mathrm{~T}$ & reproducible \\
\hline ТВ04 & TB96 A4130:2/HS1 & bone & 1996 & $\begin{array}{l}\text { phase } 11 \text {, mid } 4 \text { th millennium } \\
\text { (Middle Uruk) }\end{array}$ & 2 & no & no & - & - & - \\
\hline
\end{tabular}


Table 2 (continued)

\begin{tabular}{|c|c|c|c|c|c|c|c|c|c|c|}
\hline \multirow[t]{2}{*}{ Lab code } & \multirow[t]{2}{*}{ Achaeological code } & \multirow[t]{2}{*}{ Skeletal pan } & \multirow{2}{*}{$\begin{array}{l}\text { Date } \\
\text { excavated }\end{array}$} & \multirow[t]{2}{*}{ Extra information } & \multicolumn{4}{|c|}{ PCR amplification } & \multirow{2}{*}{$\begin{array}{l}\text { Sequence } \\
\text { cluster(s) }\end{array}$} & \multirow{2}{*}{$\begin{array}{l}\text { Reasons for } \\
\text { success/failure }\end{array}$} \\
\hline & & & & & $\begin{array}{l}\text { No. of } \\
\text { extractions }\end{array}$ & $\begin{array}{l}157 \mathrm{bp} \\
\text { product }\end{array}$ & $\begin{array}{l}176 \mathrm{bp} \\
\text { product }\end{array}$ & $\begin{array}{l}\text { Maximum } \\
\text { sequence } \\
\text { length } \\
\text { (bp) }\end{array}$ & & \\
\hline ТВ05 & TB94 A1023:2/HS & bone & 1994 & 4th-3rd millennium & 2 & 2 of 4 & no & 157 & $\mathrm{~T} / \mathrm{T} 3$ & $\begin{array}{l}\text { PCR product } \\
\text { from one extract } \\
\text { only }\end{array}$ \\
\hline ТВ06 & TB95 A6035:2/HS4 & vertebra & 1995 & $\begin{array}{l}\text { phases III/IV, late } 4 \text { th-late } 3 \mathrm{rd} \\
\text { millennium }\end{array}$ & 2 & 3 of 4 & no & 157 & $\mathrm{~T} / \mathrm{T} 3$ & $\begin{array}{l}\text { sequence mosaic } \\
\text { in one extract }\end{array}$ \\
\hline ТВ07 & TB95 A1136:2/HS3 & vertebra & 1995 & phase IV, late 3rd millennium & 2 & 4 of 4 & no & 157 & T/T3 & reproducible \\
\hline ТВ08 & TB95 A6517:3/HS4 & bone & 1995 & $\begin{array}{l}\text { phases III/IV, late } 4 \text { th-late } 3 \text { rd } \\
\text { millennium }\end{array}$ & 2 & 2 of 4 & no & 157 & $\mathrm{~T} / \mathrm{T} 3$ & contradiction \\
\hline TR10 & TB96 A4083:2/HS1 & bone & 1996 & $\begin{array}{l}\text { phase II, mid 4th millennium (Middle } \\
\text { Uruk) }\end{array}$ & 2 & 1 of 4 & no & 115 & $\mathrm{~T} 2$ & non-reproducible \\
\hline TB11 & TB95 A756:2/HS6 & vertebra & 1995 & $\begin{array}{l}\text { phase I, early-mid 4th millennium } \\
\text { (early-mid Uruk) }\end{array}$ & 2 & no & no & - & - & - \\
\hline TB12 & TB95 A155:2/HF2 & bone & 1995 & $\begin{array}{l}\text { phase III, late } 4 \text { th-early } 3 \mathrm{rd} \\
\text { millennium (Ninevite V) }\end{array}$ & 1 & no & no & - & - & - \\
\hline TB13 & TB95 A9004:2/HF2 & bone & 1995 & $\begin{array}{l}\text { phase III, late } 4 \text { th-early } 3 \mathrm{rd} \\
\text { millennium (Ninevite V) }\end{array}$ & 1 & no & no & - & - & - \\
\hline TB14 & TB?? Trench HL & bone & - & $\begin{array}{l}\text { phase III, late } 4 \text { th-early } 3 \mathrm{rd} \\
\text { millennium (Ninevite V) }\end{array}$ & 1 & no & no & - & - & - \\
\hline TB15 & TB94 A1023:12/HS3A & bone & 1994 & phase IV, late 3rd millennium & 1 & no & no & - & - & - \\
\hline TB16 & TB94 A1023:12/H83B & bone & 1994 & phase IV, late 3 rd millennium & 2 & 3 of 4 & no & 157 & $\mathrm{~T} / \mathrm{T} 3$ & contradiction \\
\hline TB17 & TB94 A62:3/HN & bone & 1994 & phase V, 2nd millennium & 1 & no & no & - & - & - \\
\hline TB18 & TB96 A244:2/HN & bone & 1996 & phase $\mathrm{V}$, 2nd millennium & 1 & no & no & - & - & - \\
\hline TB19 & TB96 A264:10/HN & bone & 1996 & phase V, 2nd millennium & 1 & no & no & - & - & - \\
\hline TB20 & TB94 A1023:12/HS3C & vertebra & 1994 & phase IV, late 3rd millennium & 1 & no & no & - & - & - \\
\hline TB21 & TB96 A252:2/HN & limb bone & 1996 & phase $\mathrm{V}$, 2nd millennium & 2 & 1 of 4 & no & 157 & $\mathrm{~T} / \mathrm{T} 3$ & non-reproducible \\
\hline TB22 & TB96 A215:2/HN & vertebra & 1996 & $\begin{array}{l}\text { phase } \mathrm{V}, \text { 2nd millennium; possibly } B \text {. } \\
\text { indicus }\end{array}$ & 2 & 1 of 4 & no & 157 & $\mathrm{~T} / \mathrm{T} 3$ & non-reproducible \\
\hline TB23 & TB96 A192:2/HN & bone & 1996 & $\begin{array}{l}\text { phase } \mathrm{V}, \text { 2nd millennium; possibly } B \text {. } \\
\text { indicus }\end{array}$ & 2 & 1 of 4 & no & 157 & $\mathrm{~T} / \mathrm{T} 3$ & non-reproducible \\
\hline ТВ24 & TB96 A4165:2/HS1 & limb bone & 1996 & $\begin{array}{l}\text { phase II, mid 4th millennium (Middle } \\
\text { Uruk) }\end{array}$ & 1 & no & no & - & - & - \\
\hline \multicolumn{11}{|c|}{ Ardèche-c. $7000-7500$ cal BP (early Neolithic) $[38,39]$} \\
\hline $\begin{array}{l}\text { Ardèche } \\
01\end{array}$ & $\begin{array}{l}\text { Baume d'Oullen-BA66 } \\
\text { ADN; BO83-N12.C46 } \\
\text { Z.25 }\end{array}$ & tooth & 1983 & $\begin{array}{l}\text { early Cardial Neolithic }(6630 \pm 110 \\
\text { bp); karstic cave site }\end{array}$ & 2 & 2 of 2 & no & 129 & $\mathrm{~T} / \mathrm{T} 3$ & reproducible \\
\hline $\begin{array}{l}\text { Ardèche } \\
02\end{array}$ & $\begin{array}{l}\text { Combe Obscure-CO74 } \\
\text { G4 che } 637\end{array}$ & jaw bone & 1974 & $\begin{array}{l}\text { Cardial Neolithic (earlier than } \\
6400 \pm 160 \text { bp); karstic cave site }\end{array}$ & 2 & 2 of 2 & no & 129 & $\mathrm{~T} / \mathrm{T} 3$ & reproducible \\
\hline
\end{tabular}




\begin{tabular}{|c|c|c|c|c|c|c|c|c|c|c|}
\hline \multirow[t]{2}{*}{ Lab code } & \multirow[t]{2}{*}{ Achaeological code } & \multirow[t]{2}{*}{ Skeletal pan } & \multirow{2}{*}{$\begin{array}{l}\text { Date } \\
\text { excavated }\end{array}$} & \multirow[t]{2}{*}{ Extra information } & \multicolumn{4}{|c|}{ PCR amplification } & \multirow{2}{*}{$\begin{array}{l}\text { Sequence } \\
\text { cluster(s) }\end{array}$} & \multirow{2}{*}{$\begin{array}{l}\text { Reasons for } \\
\text { success/failure }\end{array}$} \\
\hline & & & & & $\begin{array}{l}\text { No. of } \\
\text { extractions }\end{array}$ & $\begin{array}{l}157 \mathrm{bp} \\
\text { product }\end{array}$ & $\begin{array}{l}176 \mathrm{bp} \\
\text { product }\end{array}$ & $\begin{array}{l}\text { Maximum } \\
\text { sequence } \\
\text { length } \\
\text { (bp) }\end{array}$ & & \\
\hline $\begin{array}{l}\text { Ardèche } \\
03\end{array}$ & $\begin{array}{l}\text { Combe Obscure-CO74 } \\
\text { H5 chc } 6 \text { A } 264\end{array}$ & jaw bone & 1974 & $\begin{array}{l}\text { Cardial Neolithic (earlier than } \\
6400 \pm 160 \text { bp); karstic cave site }\end{array}$ & 2 & no & no & - & - & - \\
\hline \multicolumn{11}{|c|}{ Bercy, Paris - c. 5000 cal BP (middle Neolithic) $[3,5,46]$} \\
\hline Bercy 01 & $\begin{array}{l}\text { QS. MXVII 16-C21; MB } \\
10000\end{array}$ & right & - & $\begin{array}{l}\text { upper part of river bank; good } \\
\text { collagen content }\end{array}$ & 2 & no & no & - & - & inhibition present \\
\hline Bercy 02 & QS. MXVI 24; MB 20000 & $\begin{array}{l}\text { right } \\
\text { mandible }\end{array}$ & - & $\begin{array}{l}\text { upper part of river bank; good } \\
\text { collagen content }\end{array}$ & 2 & no & no & - & - & - \\
\hline Bercy 03 & QS. MXV 8; MB 21000 & $\begin{array}{l}\text { right } \\
\text { mandible }\end{array}$ & - & $\begin{array}{l}\text { upper part of river bank; good } \\
\text { collagen content }\end{array}$ & 2 & no & no & - & - & - \\
\hline Bercy 04 & QS. MXV, MB 23000 & $\begin{array}{l}\text { right } \\
\text { mandible }\end{array}$ & - & $\begin{array}{l}\text { upper part of river bank; good } \\
\text { collagen content }\end{array}$ & 2 & no & no & - & - & - \\
\hline Bercy 05 & $\begin{array}{l}\text { QS. LXII 9-C21; MB } \\
24000\end{array}$ & $\begin{array}{l}\text { right } \\
\text { mandible }\end{array}$ & - & $\begin{array}{l}\text { lower part of river bank; low } \\
\text { collagen content }\end{array}$ & 2 & no & no & - & - & inhibition present \\
\hline Bercy 06 & $\begin{array}{l}\text { QS. MXVIII 13-C21; MB } \\
28000\end{array}$ & $\begin{array}{l}\text { right } \\
\text { mandible }\end{array}$ & - & $\begin{array}{l}\text { upper part of river bank; good } \\
\text { collagen content }\end{array}$ & 2 & no & no & - & - & - \\
\hline Bercy 07 & $\begin{array}{l}\text { QS. MXX 22-C21; MB } \\
29000\end{array}$ & $\begin{array}{l}\text { right } \\
\text { mandible }\end{array}$ & - & $\begin{array}{l}\text { upper part of river bank; good } \\
\text { collagen content }\end{array}$ & 2 & no & no & - & - & - \\
\hline Bercy 08 & $\begin{array}{l}\text { QS. MXX 11-C21; MB } \\
33000\end{array}$ & $\begin{array}{l}\text { right } \\
\text { mandible }\end{array}$ & - & $\begin{array}{l}\text { upper part of river bank; good } \\
\text { collagen content }\end{array}$ & 2 & no & no & - & - & - \\
\hline Bercy 09 & $\begin{array}{l}\text { QS. MXV 21-C21; MB } \\
35000\end{array}$ & $\begin{array}{l}\text { right } \\
\text { mandible }\end{array}$ & - & $\begin{array}{l}\text { upper part of river bank; good } \\
\text { collagen content }\end{array}$ & 2 & 2 of 2 & no & 157 & $\mathrm{~T} / \mathrm{T} 3$ & reproducible \\
\hline Bercy 10 & QS. MXV; MB 71000 & $\begin{array}{l}\text { right } \\
\text { mandible }\end{array}$ & - & $\begin{array}{l}\text { upper part of river bank; good } \\
\text { collagen content }\end{array}$ & 2 & 2 of 2 & no & 117 & $\mathrm{~T} 2$ & reproducible \\
\hline Bercy 11 & $\begin{array}{l}\text { QS. LXV 9-C21; ME } \\
79000\end{array}$ & $\begin{array}{l}\text { right } \\
\text { mandible }\end{array}$ & - & $\begin{array}{l}\text { lower part of river bank; good } \\
\text { collagen content }\end{array}$ & 2 & no & no & - & - & - \\
\hline Bercy 12 & $\begin{array}{l}\text { QS. LXX 11-C21a; MB } \\
86000\end{array}$ & $\begin{array}{l}\text { right } \\
\text { mandible }\end{array}$ & - & $\begin{array}{l}\text { lower part of river bank; good } \\
\text { collagen content }\end{array}$ & 2 & no & no & - & - & inhibition present \\
\hline Bercy 13 & $\begin{array}{l}\text { QS. MX13-C21; MB } \\
89000\end{array}$ & $\begin{array}{l}\text { right } \\
\text { mandible }\end{array}$ & - & $\begin{array}{l}\text { upper part of river bank; good } \\
\text { collagen content }\end{array}$ & 2 & 2 of 2 & no & 129 & $\mathrm{~T} 3$ & reproducible \\
\hline \multicolumn{11}{|c|}{ West Africa-c. 2200-600 BP (Iron Age) [28] } \\
\hline Africa 01 & $\begin{array}{l}\text { Jenné-jeno, Mali-Jj85 } \\
\text { LXN L52 }\end{array}$ & bone & - & $\begin{array}{l}\text { located under layer radiocarbon } \\
\text { dated to } 2090 \pm 110 \mathrm{bp}\end{array}$ & 2 & no & no & - & - & - \\
\hline Africa 02 & $\begin{array}{l}\text { Kariana, Mali-Jj81 } \\
\text { KAN L2 }\end{array}$ & bone & - & dating to $\mathrm{c} . \mathrm{AD} 1000-1200$ & 2 & no & no & - & - & - \\
\hline Africa 03 & $\begin{array}{l}\text { Sincu Bars, } \\
\text { Senegal-SBAI L12 }\end{array}$ & bone & - & dating to c. AD $700-1000$ & 2 & no & no & - & - & - \\
\hline
\end{tabular}


Table 2 (continued)

\begin{tabular}{|c|c|c|c|c|c|c|c|c|c|c|}
\hline \multirow[t]{2}{*}{ Lab code } & \multirow[t]{2}{*}{ Achaeological code } & \multirow[t]{2}{*}{ Skeletal pan } & \multirow{2}{*}{$\begin{array}{l}\text { Date } \\
\text { excavated }\end{array}$} & \multirow[t]{2}{*}{ Extra information } & \multicolumn{4}{|c|}{ PCR amplification } & \multirow{2}{*}{$\begin{array}{l}\text { Sequence } \\
\text { cluster(s) }\end{array}$} & \multirow{2}{*}{$\begin{array}{l}\text { Reasons for } \\
\text { success/failure }\end{array}$} \\
\hline & & & & & $\begin{array}{l}\text { No. of } \\
\text { extractions }\end{array}$ & $\begin{array}{l}157 \mathrm{bp} \\
\text { product }\end{array}$ & $\begin{array}{l}176 \mathrm{bp} \\
\text { product }\end{array}$ & $\begin{array}{l}\text { Maximum } \\
\text { sequence } \\
\text { length } \\
\text { (bp) }\end{array}$ & & \\
\hline Africa $04 a$ & $\begin{array}{l}\text { Jenné-jeno, } \\
\text { Mali-Jj77M1-20 }\end{array}$ & bone & - & dating to c. $\mathrm{AD} 400-600$ & 2 & no & no & - & - & - \\
\hline Africa $04 b$ & $\begin{array}{l}\text { Jenné-jeno, } \\
\text { Mali-Jj77M1-20 }\end{array}$ & bone & - & dating to c. AD $400-600$; burnt bone & 2 & no & no & - & - & - \\
\hline Africa $05 \mathrm{a}$ & $\begin{array}{l}\text { Jenné-jeno, Mali-Jj81 } \\
\text { LXN L52 }\end{array}$ & bone & - & $\begin{array}{l}\text { located under layer radiocarbon } \\
\text { dated to } 2090 \pm 110 \mathrm{bp}\end{array}$ & 2 & no & no & - & - & - \\
\hline Africa $05 b$ & $\begin{array}{l}\text { Jenné-jeno, Mali-Jj81 } \\
\text { LXN L52 }\end{array}$ & bone & - & $\begin{array}{l}\text { located under layer radiocarbon } \\
\text { dated to } 2090 \pm 110 \mathrm{bp}\end{array}$ & 2 & no & no & - & - & - \\
\hline Africa $05 \mathrm{c}$ & $\begin{array}{l}\text { Jenné-jeno, Mali-Jj81 } \\
\text { LXN L52 }\end{array}$ & bone & - & $\begin{array}{l}\text { located under layer radiocarbon } \\
\text { dated to } 2090 \pm 110 \mathrm{bp}\end{array}$ & 2 & 2 of 2 & no & 117 & $\mathrm{~T} 1$ & reproducible \\
\hline Africa $05 \mathrm{~d}$ & $\begin{array}{l}\text { Jenné-jeno, Mali-Jj81 } \\
\text { LXN L52 }\end{array}$ & bone & - & $\begin{array}{l}\text { located under layer radiocarbon } \\
\text { dated to } 2090 \pm 110 \mathrm{bp}\end{array}$ & 2 & 2 of 2 & no & 157 & $\mathrm{~T} 1$ & reproducible \\
\hline Africa 06 & $\begin{array}{l}\text { Jenné-jeno, Mali-Jj81 } \\
\text { LXN L31 }\end{array}$ & bone & - & dating to c. $\mathrm{AD} 850-1100$ & 2 & 2 of 2 & no & 117 & $\mathrm{~T} / \mathrm{T} 3$ & reproducible \\
\hline Africa 07 & $\begin{array}{l}\text { Jenné-jeno, Mali-Jj77 } \\
\text { M1-15 }\end{array}$ & bone & - & dating to c. $\mathrm{AD} 600-850$ & 2 & 2 of 2 & no & 157 & $\mathrm{~T} 1$ & reproducible \\
\hline Africa 08 & $\begin{array}{l}\text { Jenné-jeno, Mali-Jj77 } \\
\text { M1-5 }\end{array}$ & bone & - & dating to $\mathrm{c}$. AD $1100-1400$ & 2 & no & no & - & - & - \\
\hline Africa $09 a$ & $\begin{array}{l}\text { Jenné-jeno, Mali-Jj77 } \\
\text { M1-8 }\end{array}$ & bone & - & dating to c. AD $1100-1400$ & 2 & no & no & - & - & - \\
\hline Africa $09 b$ & $\begin{array}{l}\text { Jenné-jeno, Mali-Jj77 } \\
\text { M1-8 }\end{array}$ & bone & - & dating to c. AD $1100-1400$ & 2 & no & no & - & - & - \\
\hline Africa 10 & $\begin{array}{l}\text { Jenné-jeno, Mali-Jj8 } \\
\text { LXN L47 }\end{array}$ & bone & - & $\begin{array}{l}\text { situated above a C14 sample dated } \\
1910 \pm 110 \mathrm{bp}\end{array}$ & 2 & no & no & - & - & - \\
\hline Afica 11 & $\begin{array}{l}\text { Jenné-jeno, Mali-Jj8J } \\
\text { LXS L48 }\end{array}$ & bone & - & dating to c. AD $1100-1400$ & 2 & no & no & - & - & - \\
\hline
\end{tabular}

Archaeological cattle samples studied, with associated codes, skeletal element used, date excavated and extra information supplied. Site details and dates are given above each set of specimens. Archaeological dates are based on the context of, and evidence from, each excavation. PCR amplification results and maximum mitochondrial region sequence lengths are included. The last two columns indicate the B. taurus cluster(s) to which each sequence belongs (see text), and reasons for success or failure. N/a, not known or not applicable; non-reproducible, only one sequence obtained from several attempts; contradiction, two or more sequences obtained, but results different; reproducible, at least three reproducible sequences from two different extractions; $n d$, not done. 
materials [3]. The African bones were, on the whole, dense and dark brown in colour, with much trapped loose soil within the bone cavities.

\subsection{DNA extraction and amplification of archaeological specimens}

Bone samples were prepared using the procedure described by MacHugh et al. [31] that was modified from Yang et al. [50]. Before extraction, each bone or tooth was sandblasted to remove external contamination that can out-compete endogenous DNA [37]. The numbers of DNA extractions performed for each specimen are indicated in Table 2. In the cases where only one extract was attempted, this was because no amplification products were obtained from several attempts using the first extract.

Polymerase chain reaction (PCR) set-up was conducted in a laboratory dedicated solely to preamplification ancient work. PCR conditions were as described in MacHugh et al. [30]. The region analysed was a defined, highly variable region of the mtDNA control region between bases 16,022 and 16,262 [27]. Amplification was attempted for both a 157 base pair fragment, $\mathrm{AN} 2_{\mathrm{FOR}}(16,022-16,041)-\mathrm{AN} 1_{\mathrm{REV}}(16,178-$ $16,159)$; and a 176 bp region, $\mathrm{AN} 1_{\mathrm{FOR}}(16,159$ $16,178)-\mathrm{AN} 3_{\mathrm{REV}}(16,334-16,314)$. These primers are species-specific so do not amplify human DNA. Direct sequencing procedures were carried out as described in detail by MacHugh et al. [31].

Second-round PCR was not undertaken on any samples that did not amplify in the first-round. All non-amplifiable samples were tested for presence of inhibitors that may have been impeding PCR amplification of endogenous DNA. This involved spiking each negative sample extract with aurochs DNA (from 5000 BP Carsington Pasture Cave) in a ratio of 1:4. PCR was continued as previously described, and those samples that yielded a product from the spiked sample were then designated as containing little or no endogenous DNA.

The criteria for authenticating mitochondrial haplotypes were as follows. For working samples, at least two independent extractions were undertaken from those samples that produced amplifiable DNA (Table 2). Subsequently, a range of PCR amplifications and subsequent direct sequencing analyses were performed from each purified DNA extract. Reproducible samples were designated as those that gave consistent sequences in three or more amplifications, with at least one from each of two separate extractions. In order to be considered authentic, any mutations observed had to be replicated in sequences from two separate extracts. The mtDNA sequences thus derived from each sample were therefore verified through independent extractions, amplifications and sequence determinations.

\subsection{Statistical and phylogenetic analysis}

mtDNA sequences were aligned by eye (Table 1), and reduced median networks (Fig. 1) were constructed using the median algorithm of Bandelt et al. [4]. The networks include published data from the following regions: Anatolia (43), Middle East (37), Mainland Europe (91), and Africa (95). This data comprises that analysed by Troy et al. [47], truncated to the $117 \mathrm{bp}$ presented in this paper. In Fig. 1, all sequences are shaded corresponding to which of the five B. taurus haplogroups they group with (T, T1, T2, T3 or T4). The skeleton network estimates the phylogenetic relationship between these five haplogroups and the British B. primigenius reference sequence (GENBANK accession number AF336746; [47]). As can be seen from Table 1, the segment amplified in the ancient sequences does not contain the 16,225 base pair change that differentiates $\mathrm{T}$ from T3 (as designated by Troy et al. [47]), and therefore these two haplogroups have been amalgamated into the $\mathrm{T} / \mathrm{T} 3$ group discussed here.

Along with other regional data (32 Japanese, 65 British and 51 Western European Fringe B. taurus; $[33,47])$, the truncated modern data was included in analyses of inter-population genetic distances between extant and ancient populations. Linearised $F_{\mathrm{ST}}$ values, with associated $P$-values after 1023 permutations, were generated (Table 3) according to Slatkin [43] using the ARLEQUIN computer program (version 2.000 [42]). The $P$-value of the test is the proportion of permutations leading to a $F_{\mathrm{ST}}$ value larger or equal to the observed one, therefore low $P$-values equate to significant genetic distances. The ancient sequences were regionally grouped. The neighbor-joining method [41] was used to construct a dendrogram of breed relationships from the genetic distances (Fig. 2) using the PHYLIP package [11].

\section{Results}

\subsection{Overall amplification success}

Table 2 gives full data for amplification success in all 101 samples tested. Reproducible sequences were only obtained from 12 samples: one Çatalhöyük, two Tell Brak, one Baume d'Oullen, one Combe Obscure, three Bercy and four Jenné-jeno (Tables 1 and 2). These comprise a total of six haplotypes, differentiated by eight polymorphic sites, three of which have not been previously described. They were placed on reduced modern networks (Fig. 1). Sites prone to repeated mutations have previously been identified [47], and most of the reductions (11 sites out of 13) were at these sites.

Only one bone, from Çatalhöyük, gave amplification products with the larger $176 \mathrm{bp}$ fragment. Apart from the 12 samples that gave reproducible sequences, varying numbers of amplification products for the smaller 

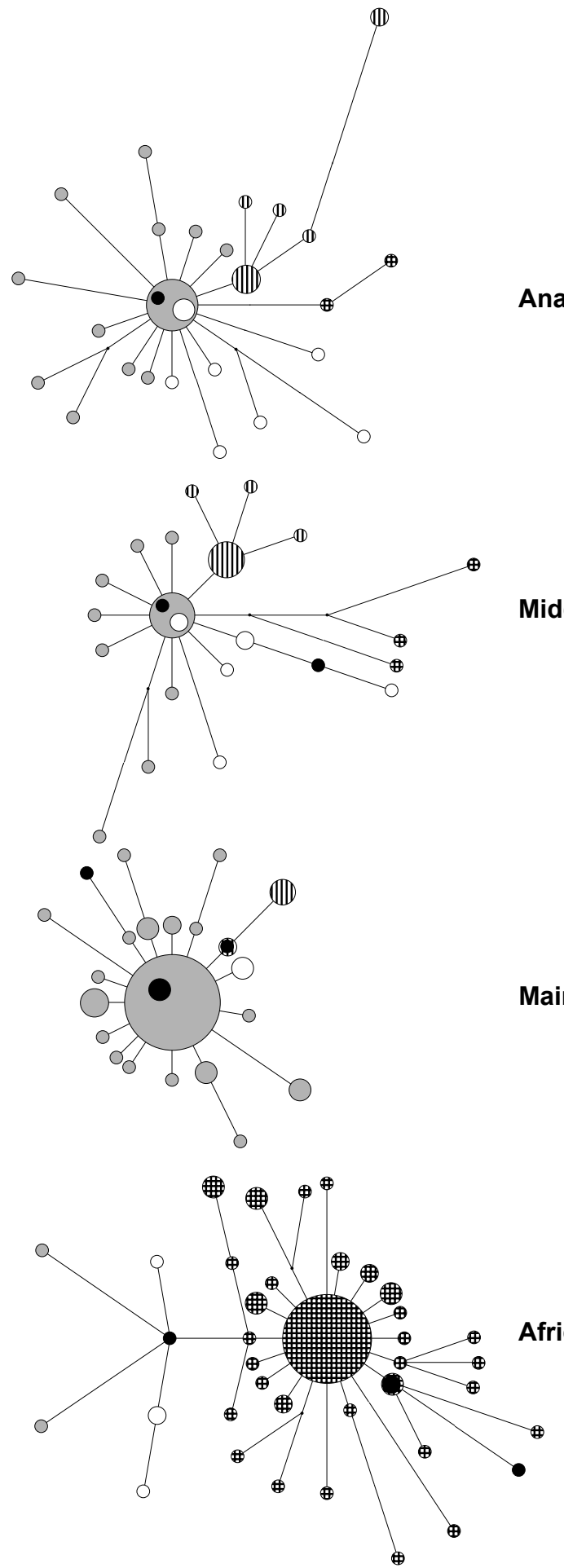

Anatolia

\section{Middle East}

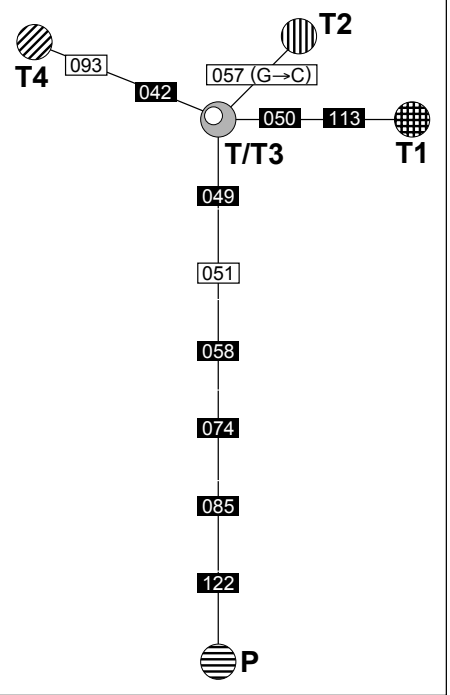

\section{Mainland Europe}

Africa 
Table 3

Linearised pairwise $F_{\mathrm{ST}}$ values of ancient and extant populations

\begin{tabular}{|c|c|c|c|c|c|c|c|c|c|c|}
\hline & $\begin{array}{l}\text { Ancient } \\
\text { Near East }\end{array}$ & $\begin{array}{l}\text { Ancient } \\
\text { Europe }\end{array}$ & $\begin{array}{l}\text { Ancient } \\
\text { Africa }\end{array}$ & Anatolia & $\begin{array}{l}\text { Middle } \\
\text { East }\end{array}$ & $\begin{array}{l}\text { Mainland } \\
\text { Europe }\end{array}$ & Britain & $\begin{array}{l}\text { Western } \\
\text { European } \\
\text { Fringe }\end{array}$ & Africa & Japan \\
\hline Ancient Near East & - & 0.0083 & 0.3812 & 0.8516 & 0.7334 & 0.3516 & 0.7920 & 0.3994 & 0.0000 & 0.0801 \\
\hline Ancient Europe & 0.6377 & - & 0.8748 & 0.9326 & 0.7246 & 0.5254 & 0.7197 & 0.3076 & 0.0000 & 0.0234 \\
\hline Ancient Africa & 0.1172 & 0.0479 & - & 0.0000 & 0.0010 & 0.0000 & 0.0000 & 0.0000 & 0.0059 & 0.0020 \\
\hline Anatolia & 0.0000 & 0.0000 & 0.5427 & - & 0.7490 & 0.0000 & 0.0117 & 0.0000 & 0.0000 & 0.0000 \\
\hline Middle East & 0.0000 & 0.0000 & 0.5388 & 0.0000 & - & 0.0000 & 0.0088 & 0.0000 & 0.0000 & 0.0000 \\
\hline Mainland Europe & 0.0337 & 0.0000 & 1.5395 & 0.0319 & 0.0616 & - & 0.0178 & 0.0310 & 1.2529 & 0.0000 \\
\hline Britain & 0.0000 & 0.0000 & 0.5988 & 0.0162 & 0.0219 & 0.0000 & - & 0.0203 & 0.7875 & 0.0000 \\
\hline $\begin{array}{l}\text { Western European } \\
\text { Fringe }\end{array}$ & 0.0000 & 0.0218 & 1.3613 & 0.0440 & 0.0839 & 0.0000 & 0.0010 & - & 1.1461 & 0.0000 \\
\hline Africa & 0.6216 & 0.9837 & 0.2709 & 0.7765 & 0.7179 & 0.0000 & 0.0000 & 0.0000 & - & 0.0000 \\
\hline Japan & 0.2209 & 0.2591 & 1.0127 & 0.2790 & 0.3049 & 0.4436 & 0.2397 & 0.3225 & 1.2098 & - \\
\hline
\end{tabular}

Population linearised $F_{\mathrm{ST}}$ values (below diagonal), with $P$-values after 1023 permutations (above diagonal), calculated using Slatkin [43]. The ancient Near East group contains the sequences TB03, TB07 and CH11; the ancient Europe sample includes Ardèche 01 and 02 , Bercy 09, Bercy 10 and Bercy 13; and the ancient African group contains Africa 05c, Africa 05d, Africa 06 and Africa 07.

$157 \mathrm{bp}$ region were obtained in a further 18 of the 101 samples amplified: eight Çatalhöyük, one Israel, and nine Tell Brak. Some of these 18 samples gave two or more amplicons that could be sequenced, in most cases from separate extractions. However, these were discounted because ostensibly identical sequences contained different substitutions (Table 2). The inhibition test showed that three of the 13 Bercy mandibles contained unknown substances that were inhibiting DNA amplification. This inhibition was overcome by using five-times the usual concentration of Taq polymerase.

\subsection{Amplification success - region by region, according to taphonomic contexts}

Only one reliable sequence was obtained from the 23 Çatalhöyük remains, although nine samples generated products. This is a success rate of $4.3 \%$, which decreases to $2.6 \%$ when a further 15 negative samples (D. MacHugh, unpublished data) are taken into consideration. Only one of the 23 samples from Israel gave an amplifiable product. However, this was excluded as a mosaic sequence. Analysis of a further five bones from $\mathrm{Abu}$ Gosh also proved unsuccessful (D. MacHugh, unpublished data). Of the 23 Tell Brak remains sampled, 11 gave products, of which two were reliable $(8.7 \%$ success). Two Ardèche samples gave reproducible results from the three bones sampled (66.7\%). The Bercy mandibles gave three reliable products out of a total of 13 samples $(23.1 \%)$, but three others were found to contain PCR inhibition and therefore may harbour intact aDNA. Of the 16 samples from West Africa, four samples gave reliable sequences $(25.0 \%)$. Two of these represent different skeletal elements of the same individual, in concordance with the sequence analysis.

\subsection{Phylogenetic results-region by region}

The haplogroups T/T3, T1 and T2 are geographically distributed in extant cattle (Fig. 1). The 12 reproducible sequences obtained from the ancient remains may be diagnosed as belonging to one of these three

Fig. 1. Reduced median network of ancient and extant regional groups. B. taurus mtDNA reduced median networks constructed from four regional haplotype groups, including both extant lineages (taken from [47] and the 12 ancient sequences obtained (here shown in black). Inset: the relationships of the five main $B$. taurus haplotypes, T, T1, T2, T3 and T4, to the British aurochs, B. primigenius, haplotype P (GENBANK accession number AF336746). As $\mathrm{T}$ is differentiated from the reference sequence, T3 [1], at position 16,255, and the region sequenced comprises only 117 bp of the D-loop, from 16,042 to 16,158 , the T and T3 consensus haplogroups have been reduced together in the network. T1 is defined by transitions at positions 16,050 and 16,113; T2 by a $\mathrm{G}$ to $\mathrm{C}$ transversion at 16,057; and T4 (a haplogroup so far only found in Japan; [33] by transitions at 16,042 and 16,093. The spatial arrangement of the skeleton network and the shading codes are preserved in the full data networks. The shading indicates which of the four $\mathrm{T}$ haplogroups each ancient sample roots to: white=T ancestral; checked=T1 African; horizontally stippled=T2 Middle Eastern/European; and light grey=T3 Middle Eastern/European. The modern data are grouped as originating in Anatolia, the Middle East, mainland Europe or Africa. Circles represent sequence haplotypes, the area being proportional to the frequency of the haplotypes. Points are theoretical intermediate nodes introduced by the median algorithm, and branches between haplotypes represent mutations. The networks were reduced at the following positions; Anatolian sequences: 16,050, 16,057, 16,074, 16,110, 16,113 and 16,138; Middle Eastern sequences: 16,049, $16,050,16,058,16,074,16,085,16,113,16,121$ and 16,122; mainland European sequences: $16, \overline{110}$ only; and African sequences: 16,049, 16,056, 16,057 , 16,084 and 16,122. Fast mutating sites are underlined above and placed in black boxes on the skeleton network. Despite the decreased fragment $\overline{\text { length }}$ represented, the spatial arrangements of the haplotypes have been conserved from [47]. The archaeological samples (shown in black) were placed in the different networks based on their geographic location; TB03 and TB07 (Anatolian); CH11 (Middle East); Bercy 09, Bercy 10, Bercy 13, Ardèche 01 and Ardèche 02 (Mainland Europe); and Africa 05c, Africa 05d, Africa 06 and Africa 07 (Africa). 


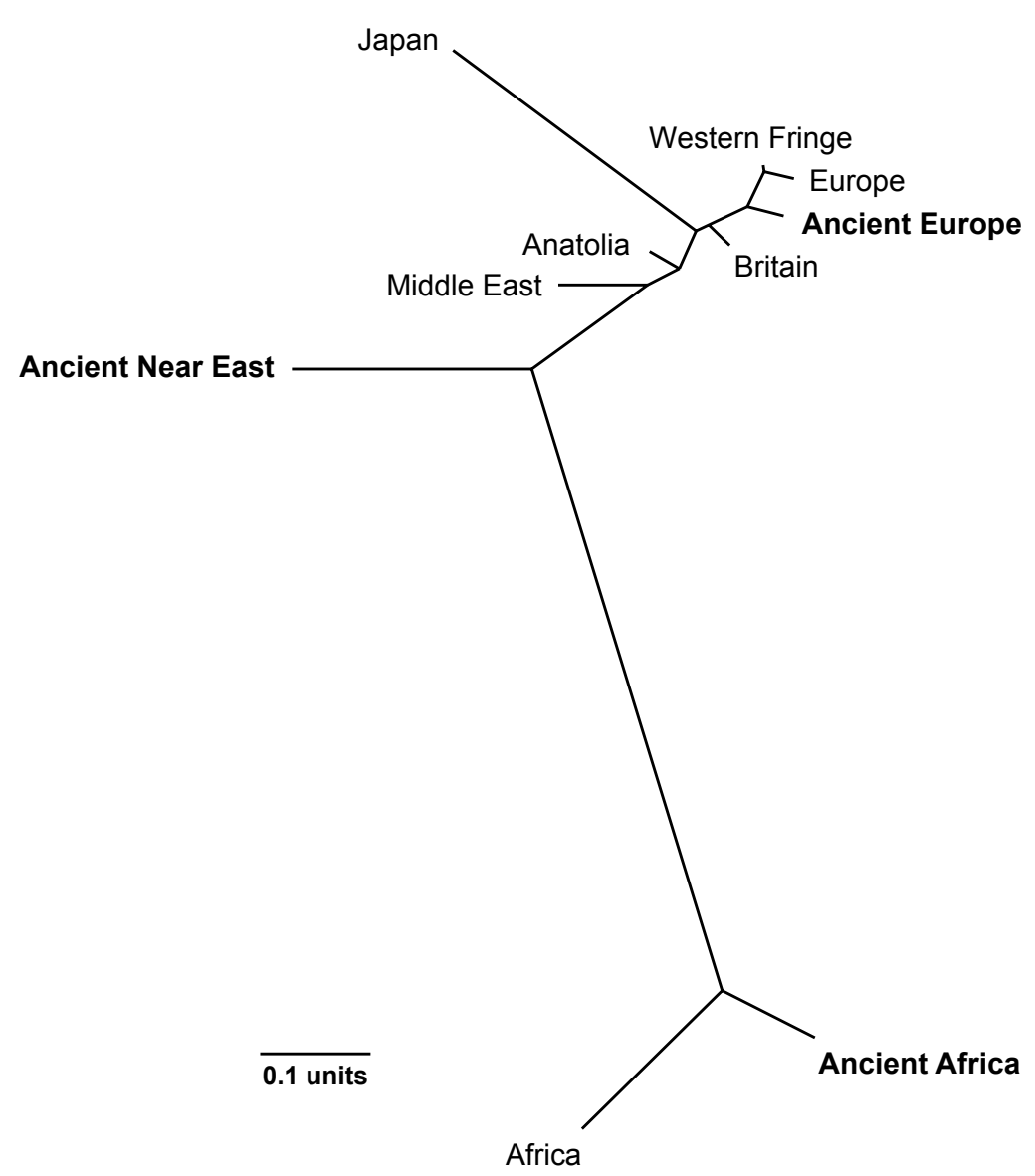

Fig. 2. Phylogenetic relationships among ancient and extant regional groups. A neighbor-joining tree summarising $F_{\mathrm{ST}}$ genetic distances between seven extant cattle groupings and three groups comprising the 12 ancient sequences obtained (see text and Table 3). The scale represents units of genetic distance and is derived from the original comparison matrix.

haplogroups (Table 2). Of the three Near Eastern sequences, two animals have the ancestral, predominant European and Near Eastern haplotype, T/T3. This group contains 15 Anatolian, 11 Middle Eastern and 54 Mainland European extant animals, as well as animals from additional regions: Britain (25), Western European Fringe (28), and Japan (4). The remaining sample from the Near East coalesces to $\mathrm{T}$, but has not previously been described. Its nearest neighbours all have $\mathrm{T}$ or $\mathrm{T} 3$ haplotypes.

Of the five reproducible French samples, both of those coming from the two Mediterranean Cardial early Neolithic sites have the same central T/T3 haplotype. Though from the same site (Bercy; middle Neolithic and northern situation), the three other samples gave three different haplotypes: a central T/T3, a previously undescribed one that coalesces to T3, and a T2, which shares a haplotype with four Anatolian and seven Middle Eastern animals, as well as one British and one Western European Fringe animal. As can be seen from the networks (Fig. 1), the T2 haplogroup is rare in mainland Europe but, as it does occur at a low frequency $(5.5 \%$ [47]), this result is not inconsistent with European variation.

One African sample has the ancestral T/T3 haplotype (Fig. 1), which is rare in extant animals from Africa (6.3\% for haplogroup T/T3 [47]). The two African samples taken from one individual are $\mathrm{T} 1$ and have the same haplotype as two other modern African sequences. The other positive sample is also T1, but this haplotype has not yet been described in extant cattle.

\subsection{Genetic diversity analyses}

Linearised $F_{\mathrm{ST}}$ values can be used as an estimate of genetic distances between populations over shallow time depths [43]. In this study, these were calculated using published mtDNA sequences, from seven geographical groups $[33,47]$ together with the 12 reliable ancient sequences, grouped into three regional populations: ancient Near East, Europe and Africa (Table 3). Both the samples from the ancient Near East and those from ancient Europe show no discernible difference to the Anatolian, Middle Eastern or British populations, but 
the associated $P$-values are high so there is a possibility that these $F_{\mathrm{ST}}$ values may have been generated by a chance allocation of haplotypes. When compared to each other, the ancient and extant African populations have a low pairwise $F_{\mathrm{ST}}$ value with a low associated $P$-value, implying a close affinity. The $F_{\mathrm{ST}}$ values between the ancient Near East population and both extant Mainland Europe and extant Africa are highly significant, as is the differentiation between extant Africa and ancient Europe.

The distance matrix was used to construct a neighbor-joining phylogeny, shown in Fig. 2. The primary feature of this tree is the major branch separating the ancient and modern African populations from the others. Both the extant Japanese and the ancient Near Eastern populations have long branches, with the latter branching most closely with the modern Anatolian and Middle Eastern populations. The European samples, ancient and extant, cluster together.

\section{Discussion}

Ancient DNA results are more readily accepted when they fit into an appropriate context. Here all reproducible sequences obtained fit within the phylogenetic framework of domesticated Near Eastern, European and African B. taurus. Also, data are consistent with regional continuity between ancient and modern cattle. Although studies of mtDNA in modern cattle have been informative in tracing the underlying patterns of European cattle domestication, the resolution of $B$. taurus mtDNA haplotypes imposes limitations on their use. The observed differences are not sufficient to differentiate between populations within regions (for example see [33]).

As with modern cattle, there appears to be no definable maternal contribution from Western European $B$. primigenius in the ancient cattle studied here, assuming sequences from six British aurochs $[2,47]$ are a representative sample of this clade. Of the 12 ancient mtDNA haplotypes obtained here, six correspond to the most common and putatively ancestral Middle Eastern and European haplotype, termed here T/T3. Apart from the three ancient samples from Africa with T1 haplotypes, which only differ from the ancestral African (T1) haplotype by up to three mutations, none of the remaining three ancient sequences differ by more than five transitions from this $\mathrm{T} / \mathrm{T} 3$ consensus.

Modern B. taurus data shows a pattern of elevated diversity in the Middle East, a subset of which is transferred to Europe, and a predominance of a different haplogroup in Africa. This pattern is followed broadly in our ancient samples. We encountered two haplotypes, $\mathrm{T}$ and $\mathrm{T} 3$, in the three samples from Çatalhöyük and Tell Brak; four of the five French samples also show $\mathrm{T}$ and $\mathrm{T} 3$ haplotypes, with the remaining one being $\mathrm{T} 2$, all of which are encountered in modern populations. On the other hand, the majority of the West African samples (three of four) fall in the modern haplogroup T1, and, although sample sizes are obviously quite small, it is interesting to note that the single occurrence of the T/T3 haplotype at Jenné-jeno dates from a period (AD 850 1100) immediately following a phase when osteometric evidence suggests increased breed diversity at the site [29]. The affinity of corresponding ancient and modern regional samples is also attested to by the proximity of these within the geographic phylogenies in Fig. 1.

In addition, although still insufficiently supported from a quantitative perspective, data in Anatolia and Europe both fit chronological and geographical trends of the general scheme of domestic diffusion as described by archaeological models (for example see [17]). Clusters on the neighbor-joining tree (Fig. 2) are organised starting from ancient Near East toward extant Middle East on the one hand, and extant/ancient Europe on the other. The branch between these two groups bears extant haplotypes of Anatolia, which is supposed to have been stocked with domestic cattle at an intermediate date between the Near East and Europe [36,49]. In France, the two haplotypes obtained from samples dated to the very beginning of the Neolithic in the Mediterranean area (Baume d'Oullen and Combe Obscure), which is suspected to derive more or less directly from Anatolia and the Near East, are both identical to those of ancient Near East (Table 1). Conversely, the three haplotypes from Bercy are all of different types, in agreement with the date of this site (later by more than two millennia) and, overall, with the supposed mosaic origin of the Middle Neolithic in the Paris Basin, resulting from both Central and Mediterranean flows [46].

In the present survey, a minority of bones contained verifiable and reproducible DNA. We believe that sporadic amplification in one replicate extraction and not in the other is most likely due to the excessive fragmentation and degradation seen in aDNA [19], particularly in poorly conserved remains, which can lead to jumping PCR [35]. Here, poorly amplifying samples often yielded products which each gave a different haplotype. This was most likely due to the presence of either a small number of copies of endogenous and/or contaminating DNA, or to the accumulation of $T a q$ polymerase errors following amplification from lowcopy number DNA [37]. The patterns of success and failure in these remains from various conditions and ages are typical, and increasing the number of extractions and amplifications could lead to spurious results as indicated by mosaicism, contradictions in sequence data obtained, and contamination problems.

It was possible to generate positive replicable amplification products from 12 of the 101 ancient samples. When a further 15 cattle remains from Çatalhöyük and 
five bones from Abu Gosh, containing no amplifiable DNA, are added to this total (D. MacHugh, unpublished data), the overall success rate drops to $9.9 \%$. The success rate varied from $0 \%$ in the sites from Israel, to two out of three in the French sites of Ardèche. This latter value is similar to success rates reported by Richards et al. [37], Bailey et al. [2], MacHugh et al. [30] and Troy et al. [47], in a range of Western European cattle and aurochs specimens dating from 450 to 12,000 BP. It also confirms the good success rate (90 out of 110 ) obtained from rabbit bones from sites in Spain and Southern France [20]. The successes generated in the Bercy and African bones, 23.1\% and 25.0\% respectively, are mirrored in the $22.2 \%$ reproducible ancient cattle samples analysed by Turner et al. [48].

Those samples taken from hotter, more arid areas gave considerably lower numbers of reproducible amplification, with Çatalhöyük having a success rate of $4.3 \%$, the sites in Israel giving no reliable products at all, and Tell Brak giving 8.7\% reproducibility. This disappointing trend has also been noted by Krings et al. [25], where mtDNA amplification in 132 human mummies and skeletons from Egypt, dating to c. 3000-2500 BP, gave only two reproducible sequences. Other aDNA analyses undertaken in hot arid climes have reported more success. Fox [12] amplified a 109 bp region of mtDNA from 15 out of 28 human Nubian bone and teeth remains from Sudan, dated 2320-2130 BP. Although this is a success rate of $53.6 \%$, only seven samples $(25 \%)$ were analysed twice. Kahila Bar-Gal et al. [23] investigated mtDNA in 20 goat bones from Abu Gosh, Israel, dating to $9500-5500 \mathrm{BP}$. Although eight bones were said to have yielded product $(40.0 \%)$, only a maximum of four $(20.0 \%)$ would be counted as reproducible using our criteria.

Samples unearthed from high temperature arid environments are less promising for archaeological genetics, which is disappointing because Anatolia and the Middle East represent the most interesting areas concerning the origins of domesticated cattle. Kumar et al. [26] reached similar conclusions when looking at human remains from open-air sites in India. It appears that analyses of archaeological DNA from hot arid sites will have to wait for the benefits that marked improvements in the technologies surrounding the extraction, amplification and analysis of degraded and damaged DNA would lend. However, all the archaeological contexts from which the samples originated, in these arid or semi-arid areas studied, were strongly submitted to weather influences, and the low success rates seen in bones from these environments appear to be in concordance with the concept that the thermal history of a sample has a significant impact on its DNA preservation [44]. In contrast, cave contexts in Mediterranean or temperate climatic areas seem to be especially favourable for DNA preservation. It would thus be necessary to test deep sedimentary contexts, such as wells or deep pits, in arid zones before being too pessimistic.

\section{Acknowledgements}

We thank Andrew Chamberlain for the CPC wild boar and aurochs specimens, and Yvonne Edwards for commenting on the manuscript. Authorisation for sampling from the various sites was from the following: E. Galili, N. Goring-Morris, H. Khalaily and O. Marder (Israel), J.-L. Roudil (Ardèche), and R. J. McIntosh (Africa). C. J. Edwards is supported by the Enterprise Ireland Basic Research Grants Scheme (project no. SC/1999/409). This work was part-funded by The Wellcome Trust (grant no. 047485/Z/96/Z), and a Eurocores OMLL Programme Grant via CNRS, France.

\section{References}

[1] S. Anderson, M.H. de Bruijn, A.R. Coulson, I.C. Eperon, F. Sanger, I.G. Young, Complete sequence of bovine mitochondrial DNA, Journal of Molecular Biology 156 (1982) 683-717.

[2] J.F. Bailey, M.B. Richards, V.A. Macaulay, I.B. Colson, I.T. James, D.G. Bradley, R.E.M. Hedges, B.C. Sykes, Ancient DNA suggests a recent expansion of European cattle from a diverse wild progenitor species, Proceedings of the Royal Society London Series B 263 (1996) 1467-1473.

[3] M. Balasse, H. Bocherens, A. Tresset, A. Mariotti, J.-D. Vigne, Émergence de la production laitière au Néolithique? Contribution de l'analyse isotopique d'ossements de bovins archéologiques, Comptes Rendus de l'Académie des Sciences de Paris. Sciences de la Terre et des Planétes 325 (1997) 1005-1010.

[4] H.-J. Bandelt, P. Forster, B.C. Sykes, M.B. Richards, Mitochondrial portraits of human populations using median networks, Genetics 141 (1995) 743-753.

[5] H. Bocherens, A. Tresset, F. Wiedemann, F. Giligny, F. Lafage, Y. Lanchon, A. Mariotti, Diagenetic evolution of mammal bones in two French Neolithic sites, Bulletin de la Société Geologique de France 168 (1997) 555-564.

[6] D.G. Bradley, D.E. MacHugh, P. Cunningham, R.T. Loftus, Mitochondrial diversity and the origins of African and European cattle, Proceedings of the National Academy of Sciences of the U.S.A. 93 (1996) 5131-5135.

[7] I.B. Colson, J.F. Bailey, M. Vercauteren, B.C. Sykes, R.E.M. Hedges, The preservation of ancient DNA and diagenesis, Ancient Biomolecules 1 (1997) 109-117.

[8] A. Cooper, H.N. Poinar, Ancient DNA: do it right or not at all, Science 289 (2000) 1139

[9] T. Cymbron, R.T. Loftus, M.I. Malheiro, D.G. Bradley, Mitochondrial sequence variation suggests an African influence in Portuguese cattle, Proceedings of the Royal Society London Series B 266 (1999) 597-603.

[10] K. Dobney, D. Jaques, W. Van Neer, Diet, economy and status: evidence from the animal bones, in: R. Matthews (Ed.), Excavations at Tell Brak Volume 4. Exploring a Regional Centre in Upper Mesopotamia, 1994-1996, McDonald Institute and British School of Archaeology in Iraq, Cambridge, in press. 
[11] J. Felsenstein, PHYLIP-Phylogeny Inference Package (Version 3.2), Cladistics 5 (1989) 164-166.

[12] C.L. Fox, mtDNA analysis in ancient Nubians supports the existence of gene flow between sub-Sahara and North Africa in the Nile Valley, Annals of Human Biology 24 (1997) 217-227.

[13] E. Galili, M. Weinstein-Evron, Prehistory and paleoenvironments of submerged sites along the Carmel coat of Israel, Paleorient 11 (1985) 37-52.

[14] E. Galili, M. Weinstein-Evron, A. Ronen, Holocene sea level changes based on submerged archaeological sites off the northern Carmel coast in Israel, Quaternary Research 29 (1988) 36-42.

[15] E. Galili, M. Weinstein-Evron, I. Hershkovitz, A. Gopher, M. Kislev, O. Lernau, L.K. Horwitz, H. Lernau, Atlit Yam: a prehistoric site on the sea floor off the Israeli coast, Journal of Field Archaeology 20 (1993) 133-157.

[16] N. Goring-Morris, The quick and the dead: The social context of aceramic Neolithic mortuary practices as seen from Kfar Hahoresh, in: I. Kuijt (Ed.), Life in Neolithic Farming Communities. Social Organization, Identity and Differentiation, Kluwer Academi, New York, 2000, pp. 103-136.

[17] J. Guilaine, De l'Orient à l'Occident: la Néolithisation de la Méditerranée. Questions ouvertes, in: La Neolitizzazione tra Oriente e Occidente, Udine, April 1999. Museo Friulino di Storia Naturale-Accademia di Udine di Scienze, Lettere e Arti, Udine, Italy, 2000, pp. 11-21.

[18] E. Hagelberg, L.S. Bell, T. Allen, A. Boyde, S.J. Jones, J.B. Clegg, Analysis of ancient bone DNA: techniques and applications, Philosophical Transactions of the Royal Society of London Series B 333 (1991) 399-407.

[19] O. Handt, M. Höss, M. Krings, S. Pääbo, Ancient DNA: methodological challenges, Experientia 50 (1994) 524-529.

[20] C. Hardy, C. Callou, J.-D. Vigne, D. Casañe, N. Dennebouy, J.-C. Mounolou, M. Monnerot, Rabbit mitochondrial DNA diversity from prehistoric to modern time, Journal of Molecular Evolution 40 (1995) 227-237.

[21] S. Haynes, A.J. Bretman, J.B. Searle, K.M. Dobney, Bone preservation and ancient DNA. The application of screening methods for predicting DNA survival, Journal of Archaeological Science 29 (2002) 585-592.

[22] I. Hodder (Ed.), Towards Reflexive Method in Archaeology: The Example at Çatalhöyük. McDonald Institute for Archaeological Research/British Institute of Archaeology at Ankara Monograph 28 (2000).

[23] G. Kahila Bar-Gal, H. Khalaily, O. Marder, P. Ducos, L.K. Horwitz, Ancient DNA evidence for the transition from wild to domestic status in Neolithic goats: a case study from the site of Abu-Gosh, Israel, Ancient Biomolecules 4 (2002) 9-17.

[24] H. Khalaily, O. Marder (Eds), The Neolithic Site of Abu Ghosh: The 1995 Excavations, Israel Antiquities Authority Reports, in press.

[25] M. Krings, A. Halim Salem, K. Bauer, H. Geisert, A.K. Malek, L. Chaix, C. Simon, D. Welsby, A. Di Rienzo, G. Utermann, A. Sajantila, S. Pääbo, M. Stoneking, mtDNA analysis of Nile River Valley populations: a genetic corridor or a barrier to migration?, American Journal of Human Genetics 64 (1999) 1166-1176.

[26] S.S. Kumar, I. Nasidze, S.R. Walimbe, M. Stoneking, Brief communication: discouraging prospects for ancient DNA from India, American Journal of Physical Anthropology 113 (2000) $129-133$.

[27] R.T. Loftus, D.E. MacHugh, D.G. Bradley, P.M. Sharp, P. Cunningham, Evidence for two independent domestications of cattle, Proceedings of the National Academy of Sciences of the USA 91 (1994) 2757-2761.

[28] K. MacDonald, Analysis of the mammalian, avian and reptilian remains, in: S.K. McIntosh (Ed.), Excavations at Jenné-jeno, Hambarketolo, and Kaniana (Inland Niger Delta, Mali), University of California Press, 1995, pp. 291-318.
[29] K. MacDonald, Invisible pastoralists: an inquiry into the origins of nomadic pastoralism in the West African Sahel, in: C. Gosden, J. Hather (Eds.), The Prehistory of Food: Appetites for Change, Routledge, London, 1999, pp. 333-349.

[30] D.E. MacHugh, C.S. Troy, F. McCormick, I. Olsaker, E. Eythrosdottir, D.G. Bradley, Early medieval cattle remains from a Scandinavian settlement in Dublin: genetic analysis and comparison with extant breeds, Philosophical Transactions of the Royal Society of London Series B 354 (1999) 99-109.

[31] D.E. MacHugh, C.J. Edwards, J.F. Bailey, D.R. Bancroft, D.G. Bradley, The extraction and analysis of ancient DNA from bone and teeth: a survey of current methodologies, Ancient Biomolecules 3 (2000) 81-102.

[32] D.A. Magee, C. Meghen, S. Harrison, C.S. Troy, T. Cymbron, C. Gaillard, A. Morrow, J.C. Maillard, D.G. Bradley, A partial African ancestry for the Creole cattle populations of the Caribbean, Journal of Heredity 93 (2002) 429-432.

[33] H. Mannen, S. Tsuji, R.T. Loftus, D.G. Bradley, Mitochondrial DNA variation and evolution of Japanese Black cattle (Bos taurus), Genetics 150 (1998) 1169-1175.

[34] L. Martin, N. Russell, D. Carruthers, Animal remains from Central Anatolian Neolithic, in: F. Gerard, L. Thissen (Eds.), The Neolithic of Central Anatolia, Yayinlari, Istanbul, 2002, pp. 193-206.

[35] S. Pääbo, D.M. Irwin, A.C. Wilson, DNA damage promotes jumping between templates during enzymatic amplification, Journal of Biological Chemistry 265 (1990) 4718-4721.

[36] J. Peters, D. Helmer, A. von den Drisch, M. Saña-Segui, Animal domestication and husbandry in PPN Northern Syria and South-Eastern Turkey, Paléorient 25 (1999) 27-47.

[37] M.B. Richards, R.E.M. Hedges, B.C. Sykes, Authenticating DNA extracted from ancient skeletal remains, Journal of Archaeological Science 22 (1995) 291-299.

[38] J.-L. Roudil, H. Saumade, La Grotte de Combe Obscure à Lagorce (Ardèche), by the authors, Montpellier.

[39] J.-L. Roudil, Le gisement néolithique de la Baume d'Oullins, Le Garn, Gard, Premières Communautés Paysannes en Méditerranée Occidentale, CNRS, Paris, 1987, pp. 523-529.

[40] N. Russell, L. Martin, Neolithic Çatalhöyük: preliminary zooarchaeological results from the renewed excavations, in: M. Mashkour, H. Buitenhuis, F. Poplin (Eds.), Archaeozoology of Southwestern Asia and Adjacent Areas 32, ARC Publicatie, Groningen, 2000, pp. 164-170.

[41] N. Saitou, M. Nei, The neighbor-joining method: a new method for reconstructing phylogenetic trees, Molecular Biology and Evolution 4 (1987) 406-425.

[42] S. Schneider, J.-M. Kueffer, D. Roessli, L. Excoffier, ARLEQUiN Version 2.000, a Software for Population Genetic Data Analysis, Genetics and Biometry Laboratory, University of Geneva, Switzerland, 1997.

[43] M. Slatkin, A measure of population subdivision based on microsatellite allele frequencies, Genetics 139 (1995) 457-462.

[44] C.I. Smith, A.T. Chamberlain, M.S. Riley, A. Cooper, C.B. Stringer, M.J. Collins, Neanderthal DNA: not just old but old and cold?, Nature 410 (2001) 771-772.

[45] W.K. Thomas, S. Pääbo, DNA sequences from old tissue remains, Methods in Enzymology 224 (1993) 406-419.

[46] A. Tresset, Early husbandry in Atlantic areas. Animal introductions, diffusions of techniques and native acculturation at the North-Western Fringe of Europe, in: J.C. Henderson (Ed.), The Prehistory and Early History of Atlantic Europe, Götebord, 1998. BAR International Series 861, Oxford, 2000, pp. 17-32.

[47] C.S. Troy, D.E. MacHugh, J.F. Bailey, D.A. Magee, R.T. Loftus, P. Cunningham, A.T. Chamberlain, B.C. Sykes, D.G. Bradley, Genetic evidence for Near-Eastern origins of European cattle, Nature 410 (2001) 1088-1091. 
[48] C.L. Turner, A. Grant, J.F. Bailey, G.A. Dover, G.W.W. Barker, Patterns of genetic diversity in extant and extinct cattle populations: evidence from sequence analysis of mitochondrial coding regions, Ancient Biomolecules 2 (1998) 235-249.

[49] J.-D. Vigne, H. Buitenhuis, Les premiers pas de la domestication animale à l'Ouest de l'Euphrate: Chypre et l'Anatolie centrale, Paléorient 25 (1999) 49-62.
[50] D.Y. Yang, B. Eng, J.S. Waye, J.C. Dudar, S.R. Saunders, Technical note: improved DNA extraction from ancient bones using silica-based spin columns, American Journal of Physical Anthropology 105 (1998) 539-543. 